\title{
INTERPOLATION, SPECTRUM ANALYSIS, ERROR-CONTROL CODING, AND FAULT-TOLERANT COMPUTING
}

\author{
José M. N. Vieira and Paulo J. S. G. Ferreira \\ Dep. de Electrónica e Telecomunicações / INESC \\ Universidade de Aveiro \\ 3810 Aveiro Portugal
}

\begin{abstract}
This paper uncovers relations between the topics mentioned in the title, relations that we believe to have gone nearly unnoticed so far. More precisely, we show that four often studied problems in signal processing, spectrum analysis, information theory, and computing are closely related or even equivalent in a certain sense (if one of them can be solved, so can any of the others, and using essentially the same algorithms). The problems are (i) a nonlinear band-limited finite-dimensional interpolation problem (ii) the problem of estimating a signal that is the superposition of a finite number of harmonics (iii) an error-control coding problem in the real field, and (iv) certain techniques that occur in algorithm-based fault tolerant computing. The advantages of recognizing these problems as equivalent are obvious: the techniques commonly used in one field can be imported to the others, the duplication of research efforts is prevented, and the overall degree of understanding of the four problems increases. New algorithms are suggested as a result of these investigations.
\end{abstract}

\section{NOTATION}

The complex $n$-dimensional space, with the usual inner product and norm, is denoted by $\mathbb{C}^{n}$. A signal is a $n$-dimensional complex vector $x$, with components, or samples, $x(0), x(1), \ldots, x(n-1)$. The Fourier matrix ${ }^{1}$ $F$ is the $n \times n$ matrix whose elements $F_{a b}$ are given by

$$
F_{a b}=e^{-\mathrm{j} \frac{2 \pi}{n} a b},
$$

where $\mathrm{j}$ denotes the imaginary unit. The discrete Fourier transform (DFT) of $x$, denoted by $\hat{x}$, is defined by $\hat{x}=F x$. A signal $x$ is band-limited if a subset of the samples of $\hat{x}$ vanish, and is low-pass if the nonzero DFT

\footnotetext{
Fax +351-34-370545, e-mails vieira@inesca.pt and pjf@inesca.pt. This work was supported by JNICT.

${ }^{1}$ To simplify the notation the usual factor $1 / \sqrt{n}$ is omitted.
}

samples are $0, \pm 1, \pm 2, \ldots, \pm m$, for some $m$ such that $2 m+1<n$.

\section{THE FOUR PROBLEMS}

The interpolation and extrapolation of finite-dimensional band-limited signals, using the Papoulis-Gerchberg iteration or similar methods, is the subject of [1-3], for example. The algorithms studied in those works operate on the entire data record, in contrast to the minimum dimension methods, which reduce the interpolation problem to a linear set of equations with as many equations as there are unknowns, and which can then be solved iteratively or noniteratively. There are two classes of such methods: the frequency-domain [4-6] and the time-domain [7-10] methods. It has been shown $[11,12]$ that these two approaches are, in a certain sense, the dual of each other.

These methods share one characteristic: the positions of the unknown samples are assumed to be known. In practice, this is not always the case, and the following problem arises.

Problem 1 To simultaneously determine the number, positions and correct amplitudes of the incorrect samples of a band-limited signal corrupted by impulsive noise.

We now consider the problem of algorithm-based fault tolerant computing, which has been addressed by several authors [13-16]. A commonly used technique is to add checksums to the data. In the real field, these checksums can be replaced by the average value of the data. Weighted checksums have also been used to extend the error-correcting capabilities. This problem is a special case of the following general problem.

Problem 2 Given a possibly corrupted subset of data samples, and a subset of the samples of a discrete orthonormal transform of the data (such as the DFT), determine the data. 
Vieira: Interpolation, spectrum analysis, error-control coding, and fault-tolerant computing

Note that the first sample of the DFT of the data

$$
\hat{x}(0)=\sum_{k=0}^{n-1} x(k)
$$

is proportional to the average of the data (the checksum). Any other DFT samples that might also be known constitute weighted checksums, that may help in determining the data after the occurrence of errors. Clearly, transforms other than the DFT might also be used for this purpose.

We now turn to the following spectrum analysis problem.

Problem 3 Given a subset of the samples of a signal, which is known to consist of a linear combination of harmonics of unknown frequencies and amplitudes,

$$
x(t)=\sum_{k=1}^{r} a_{k} e^{-\mathrm{j} 2 \pi f_{k} t}
$$

determine the signal.

This problem is well-studied, and many methods have been proposed to solve it $[17,18]$. The fourth and last problem that we consider is the following.

Problem 4 The error-control problem in the real field: devise a coding procedure capable of locating and correcting up to a certain number of errors in a finitelength block of real numbers.

A technique that is commonly used is equivalent to the following: pad the block of $k$ data words with $n-k$ zeros, and take a IDFT of length $n$. The $n$ words are then transmitted, and at the receiving end a DFT of length $n$ is taken. The $n-k$ words that were zeropadded (the syndrome) are a window over the spectrum of the error signal. The problem is how to use this information to recover the error signal itself.

\section{DISCUSSION}

The four problems just described turn out to be closely related or even equivalent. Surprisingly, these connections do not seem to be widely recognized. An exception is perhaps problem 1 and 4 - see [19] and references therein.

\subsection{Problem 1 and 2}

These are equivalent problems, as we now show. Let $x$ be the data vector, and denote by $V=\left\{i_{1}, i_{2}, \ldots, i_{p}\right\}$ the indexes of the known samples of its DFT $\hat{x}$. In problem 2 we are given the possibly corrupted data vector $x$, and the $\hat{x}\left(i_{1}\right), \hat{x}\left(i_{2}\right), \ldots, \hat{x}\left(i_{p}\right)$, whereas in problem 1 we are given just the corrupted data vector $x$. However, the hypothesis of $x$ being band-limited implies that a known subset of the samples of $\hat{x}$ has zero value. This set plays the role of $\hat{x}\left(i_{1}\right), \hat{x}\left(i_{2}\right), \ldots, \hat{x}\left(i_{p}\right)$ in problem 2 . The only difference is that, in the band-limited case, the known DFT samples are zero: $\hat{x}\left(i_{k}\right)=0$ for all $i_{k} \in V$.

\subsection{Problem 1 and 3}

These two problems are the dual of each other ${ }^{2}$. To see this, denote by $U=\left\{i_{1}, i_{2}, \ldots i_{r}\right\}$ the positions of $r$ incorrect samples of a band-limited signal $x$ with a total of $n$ samples. Let $e$ be the error signal $e=x-y$, where $y$ is the observed signal, which coincides with $x$ except for the samples whose indexes belong to $U$. Thus, $e_{k}=0$ for all $k \notin U$. Typically, $r$, the cardinal of $U$, is much less than $n$, that is, the error vector $e$ is sparse.

Let $x$ be band-limited, with $p$ zero harmonics. Then, the DFT of $y$ contains exactly $p$ samples of the DFT of $e$. For example, if $x$ is low-pass with $2 m+1$ nonzero harmonics, then the samples $m+1$ through $n-m-1$ of $y$ are equal to the corresponding samples of $e$. But then problem 1 can be rephrased as follows: given a subset of $p$ samples of the DFT $\hat{e}$ of $e$, estimate $e$. Since $\hat{e}$ is given by

$$
\hat{e}(i)=\sum_{k=1}^{r} e\left(i_{k}\right) e^{-\mathrm{j} \frac{2 \pi}{n} i_{k} i},
$$

this shows that the problem is equivalent to the problem 3 (set $\left.t=i_{k} / n\right)$, if the time and frequency domains are interchanged. We say that the two problems are the dual of each other.

\subsection{Problem 1 and 4}

The connection between problem 1 and certain topics in information theory (error-control codes in the real field) has been noted before, but does not seem to be widely known in the signal processing community. Specific algorithms have been suggested to solve this problem; we refer to the method described in [20], which is able to correct a single error, and to [19], for the correction of multiple errors.

\section{CONSEQUENCES}

The previous observations have several immediate consequences. In this summary we offer one example: a new iterative method to solve problem 1 , based on a

\footnotetext{
${ }^{2}$ The term "dual" is used here in the sense explained in $[11,12]$.
} 
discrete-discrete version of the Papoulis-Chamzas nonlinear iteration, originally proposed to solve a problem similar to problem 3 but in $L_{2}$. Since problem 1 is equivalent to problems 2 and 4 , the same algorithm can be applied to solve any of these problems.

Denote by $U=\left\{i_{1}, i_{2}, \ldots i_{t}\right\}$ the positions of $t$ incorrect samples of a band-limited signal $x$ with a total of $n$ samples. Let $e$ be the error signal $e=x-y$, where $y$ is the observed signal, which coincides with $x$ except for the samples whose indexes belong to $U$. Thus, $e_{k}=0$ for all $k \notin U$. Typically, the cardinal of $U$ is much less than $n$, that is, $e$ is unknown but sparse.

Let $x$ be band-limited, with $p$ zero harmonics. Then, the DFT of $y$ contains exactly $p$ samples of the DFT of $e$. This observation, and the sparse character of $e$, suggests the following iterative algorithm.

Initialize $\hat{g}$ with zeros (for example).

For $i=1,2, \ldots, k$ do

Insert the known samples of $\hat{e}$ into $\hat{g}$.

Inverse Fourier transform: $g=F^{-1} \hat{g}$.

Select a threshold $L$.

For $j=0,1, \ldots, n-1$ do

if $\left|g_{j}\right|<L$ then

$\left|g_{j}\right|=0$.

endif

endfor

Fourier transform: $\hat{g}=F g$.

endfor

The theoretical maximum number of errors is one half the number of redundant (known) DFT samples. Note that the total number of unknowns is $2 r$, where $r$ is the number of unknown samples, because both the indexes and values of the samples are unknown.

The performance of the algorithm is quite satisfactory under certain conditions. Generally speaking, it works very well (that is, a good approximation is available after 10 to 20 iterations) if the number of errors is substantially below the theoretical maximum, or if the errors are not contiguous, or if there is enough oversampling (redundancy). Some of these limitations are not due to the algorithm itself but to the characteristics of the problem (the band-limited interpolation problem is ill-posed when the unknown samples are contiguous $[3,3,10,21])$. Experimental evidence shows that the algorithm offers advantage when compared with the nonlinear method proposed in [19].

\section{CONCLUSION}

We examined four problems that arise in signal processing, algorithm-based fault tolerant computing, spec-

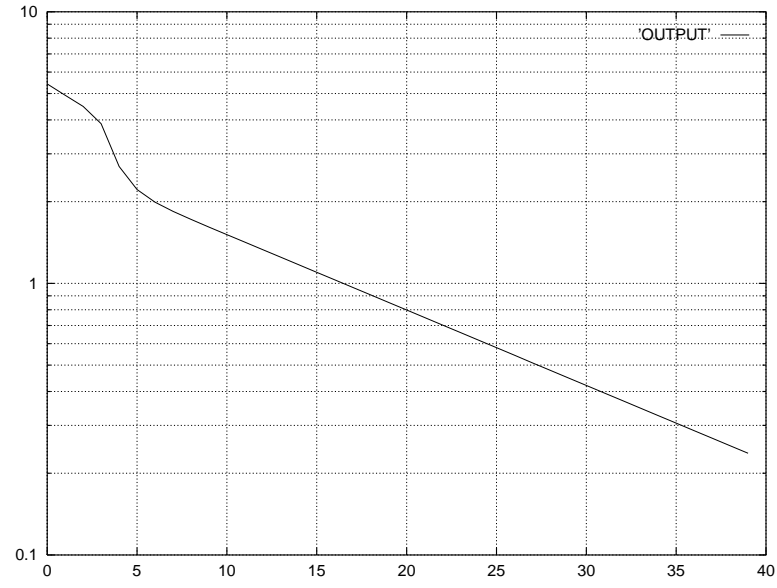

Figure 1: Error evolution plotted against the number of iterations. Interpolation of a signal with eight errors in unknown locations.

trum analysis, and information theory (error-control coding in the real field). These problems were shown to be closely related (in fact, they are either equivalent or dual). The advantages of recognizing this are obvious: the techniques commonly used in one field can be imported to the others, the duplication of research efforts is prevented, and the overall degree of understanding of the four problems increases. New algorithms were suggested based on the comparative study of these problems.

\section{REFERENCES}

[1] M. C. Jones. The discrete Gerchberg algorithm. IEEE Trans. Acoust. Speech Signal Processing, 34(3):624-626, June 1986.

[2] F. A. Marvasti. An iterative method to compensate for the interpolation distortion. IEEE Trans. Acoust. Speech Signal Processing, 37(10):16171621, October 1989.

[3] P. J. S. G. Ferreira. Interpolation and the discrete Papoulis-Gerchberg algorithm. IEEE Trans. Signal Processing, 42(10):2596-2606, October 1994.

[4] K. Gröchenig. A discrete theory of irregular sampling. Linear Algebra Appl., 193:129-150, 1993.

[5] T. Strohmer. Efficient Methods for Digital Signal and Image Reconstruction from Nonuniform Samples. PhD thesis, Institut für Mathematik der Universität Wien, November 1993.

[6] T. Strohmer. On discrete band-limited signal extrapolation. In M. E. H. Ismail, M. Z. Nashed, 
Vieira: Interpolation, spectrum analysis, error-control coding, and fault-tolerant computing

A. I. Zayed, and A. F. Ghaleb, editors, Mathematical Analysis, Wavelets, and Signal Processing, volume 190 of Contemporary Mathematics, pages 323-337, Providence, Rhode Island, 1995. American Mathematical Society.

[7] P. J. S. G. Ferreira. Noniterative and faster iterative methods for interpolation and extrapolation. IEEE Trans. Signal Processing, 42(11):3278-3282, November 1994.

[8] P. J. S. G. Ferreira and A. J. Pinho. Errorless restoration algorithms for band-limited images. In Proceedings of the First IEEE International Conference on Image Processing, ICIP-94, volume III, pages 157-161, Austin, TX, U.S.A., November 1994.

[9] P. J. S. G. Ferreira. The stability of a procedure for the recovery of lost samples in band-limited signals. Sig. Proc., 40(3):195-205, December 1994.

[10] P. J. S. G. Ferreira. The stability of certain image restoration problems: quantitative results. In Proceedings of the Second IEEE International Conference on Image Processing, ICIP-95, volume II, pages 29-32, Washington, D.C., U.S.A., October 1995.

[11] P. J. S. G. Ferreira. Interpolation in the time and frequency domains. IEEE Sig. Proc. Letters, 3(6):176-178, June 1996.

[12] P. J. S. G. Ferreira. The duality of two recent image interpolation methods. In Proceedings of the Third IEEE International Conference on Image Processing, ICIP-96, pages 735-738, Lausanne, Switzerland, September 1996.

[13] C. J. Anfinson and F. T. Luk. A linear algebraic model of algorithm-based fault tolerance. IEEE Trans. Comput., 37(12):1599-1604, December 1988 .

[14] K.-H. Huang and J. A. Abraham. Algorithmbased fault tolerance for matrix operations. IEEE Trans. Comput., 33(6):518-528, June 1984.

[15] V. S. S. Nair and J. A. Abraham. Real-number codes for fault-tolerant matrix operations on processor arrays. IEEE Trans. Comput., 39(4):426435, April 1990.

[16] A. L. Narasimha Reddy and P. Bannerjee. Algorithm-based fault detection for signal processing applications. IEEE Trans. Comput., 39(10):1304-1308, October 1990.
[17] S. M. Kay and S. L. Marple. Spectrum analysis a modern perspective. Proc. IEEE, 69(11):13801419, November 1981. Reprinted by IEEE Press, (Modern Spectrum Analysis II).

[18] A. Papoulis and C. Chamzas. Detection of hidden periodicities by adaptive extrapolation. IEEE Trans. Acoust. Speech Signal Processing, 27(5):492-500, October 1979.

[19] F. A. Marvasti and M. Nafie. Sampling theorem: A unified outlook on information theory, block and convolutional codes. IEICE Trans. Fundam. Electron. Commun. Comput. Sci., E76A(9):1383-1391, September 1993.

[20] J. K. Wolf. Redundancy, the discrete Fourier transform, and impulse noise cancellation. IEEE Trans. Commun., 31(3):458-461, March 1983.

[21] P. J. S. G. Ferreira. A class of eigenvalue problems in interpolation, extrapolation and sampling. In SampTA'95, 1995 Workshop on Sampling Theory and Applications, pages 125-136, Jurmala, Latvia, September 1995. 